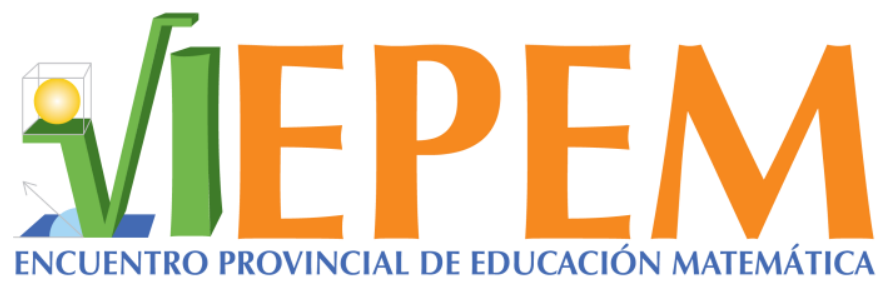

VI Encuentro Provincial de Educación Matemática.

27 al 29 de setiembre, 2017. Puntarenas, Costa Rica.

\title{
Construcción de situaciones problema que se resuelvan con modelización matemática
}

\author{
Mónica Rojas-Castro \\ monica.rojas.castro@una.cr \\ Universidad Nacional \\ Costa Rica \\ Karen Porras-Lizano \\ karen.porras.lizano@una.cr \\ Universidad Nacional \\ Costa Rica
}

\section{Resumen}

El presente taller está dirigido tanto a docentes en ejercicio como a docentes en formación; pues el principal objetivo de este es dotar a los docentes de herramientas para facilitar la construcción de situaciones problema donde se utilice modelización matemática como estrategia metodológica y que puedan ser aplicadas en el aula en línea con el Programa de Estudio en Matemática para la Educación General Básica y el Ciclo Diversificado que empezó a regir a partir del 2012. Queremos incentivar a los docentes a construir situaciones problema de calidad de manera apropiada y crítica usando los modelos matemáticos, para que sean de gran beneficio para el aprendizaje de sus estudiantes.

Palabras clave: modelización matemática; resolución de problemas; educación.

\section{Introducción}

En mayo del 2012 se aprobó en Costa Rica un currículo de Matemática que abarcaría tanto la enseñanza para primaria como para secundaria. En aras de motivar el progreso educativo, social y cultural del país, se implantó como enfoque principal la resolución de

Taller

Rojas-Castro, M. y Porras-Lizano, K. (2017). Construcción de situaciones problema que se resuelvan con modelización matemática. En Y. Morales-López, M. Picado, R. Gamboa, C. Martínez, M. Castillo y R. Hidalgo (Eds.), Memorias del VI Encuentro Provincial de Educación Matemática, Costa Rica, 2017 (pp. 31-34). Heredia: Universidad Nacional. ISBN: 978-9968-96615-3. DOI: http://dx.doi.org/10.15359/epem.6.7 
Taller dirigido a docentes de secundaria para la construcción de situaciones problema que se resuelvan con modelización matemática

problemas en contextos reales como especial énfasis. Para cumplir con lo solicitado en los programas de educación, los docentes deben buscar o crear problemas contextualizados para promover en el estudiante la construcción del conocimiento y el desarrollo de competencias matemáticas que le permitan desenvolverse en su cotidianidad.

Es importante reconocer que el cambio generó un compromiso extra por parte del gobierno, pero, especialmente, de los docentes; para esto es necesario la capacitación de estos últimos en torno al planteamiento de problemas con contextos reales que permitan al estudiante usar la matemática en diferentes situaciones de la vida social e individual.

\section{Metodología del taller}

Como primera actividad del taller se expondrán los principios fundamentales a considerar que certifican que la situación propuesta en el problema se base en situaciones de la vida real del estudiante y se diferencie de un problema tradicional carente de significado para su vida. Además, se socializarán algunos ejemplos de situaciones problema planteados según los principios expuestos. Según Porras y Fonseca (2015) "La actividad debe poseer criterios claros donde el estudiantado sea capaz de juzgar la utilidad y el progreso de las soluciones preliminares que surgen durante la resolución del problema" y que le "permitan revelar explícitamente la forma en que está pensando acerca de la situación propuesta".

En una segunda etapa, se plantearán algunos problemas que deben resolver los participantes del taller para que visualicen el proceso desde la perspectiva de los estudiantes. Además, será una forma de ejemplificar todo lo expuesto en la primera etapa y hacer una clara identificación de los principios que debe tener una actividad de modelización matemática desde la perspectiva de Lesh y Doerr (2003).

Como última etapa, se dará un espacio para que los partícipes del taller, en equipos de trabajo de tres integrantes, construyan problemas aplicando modelización matemática para los contextos a los que pertenecen sus estudiantes. Se deben exponer todos los criterios utilizados y así evaluar cada problema propuesto y regular errores y dificultades que los docentes muestren durante el taller.

En la tabla 1 se especifica la distribución del trabajo de cada actividad según las etapas descritas anteriormente. 
Taller dirigido a docentes de secundaria para la construcción de situaciones problema que se resuelvan con modelización matemática

Tabla 1

Cronograma de actividades del taller.

\begin{tabular}{lc}
\hline \multicolumn{1}{c}{ Actividad } & Tiempo \\
\hline $\begin{array}{l}\text { Exposición de principios de Lesh y Doerr para la construcción de situaciones } \\
\text { problema. }\end{array}$ & 30 minutos \\
Socialización de problemas planteados según los principios de Lesh y Doerr & 15 minutos \\
Puesta en práctica de algunos problemas socializados. & 45 minutos \\
Identificación de los principios en la actividad anterior. & 15 minutos \\
Construcción de problemas aplicando modelización matemática. & 1 hora \\
Evaluación de cada problema y regulación de errores & 30 minutos \\
Conclusiones y sugerencias & 15 minutos \\
\hline
\end{tabular}

\section{Resultados esperados}

La exposición de principios y criterios pretende generar consciencia acerca de la importancia de construir de manera apropiada problemas de calidad, para que estos cumplan su cometido de evidenciar que el trabajo basado en modelización matemática aporte al sentido de utilidad de la matemática y se estimule asertivamente al estudiantado para que apliquen el conocimiento matemático previo o logrado durante la modelización.

Además, como consecuencia del trabajo realizado por los participantes en la tercera etapa, se espera generar un banco de problemas de contextos reales contribuyendo, de esta forma, en mejorar la calidad de la educación secundaria de una parte de la comunidad educativa de nuestro país. Este banco de problemas se creará en un espacio en google drive para que todos tengan acceso mediante un enlace que se compartirá desde el inicio del taller.

\section{Referencias}

Lesh, R., y Doerr, H. M. (2003). Beyond Constructivism: Models and Modeling Perspectives on Mathematics Problem Solving, Learning, and Teaching[Más allá del constructivismo: Perspectivas sobre Modelos y Modelado en la Solución de Problemas Matemáticos, Aprendizaje y Enseñanza.]. Mahwash, New Jersey: Lawrence Erlbaum Association. 
Taller dirigido a docentes de secundaria para la construcción de situaciones problema que se resuelvan con modelización matemática

Porras, K., y Fonseca, J. (2015). Aplicación de Actividades de modelización matemática en la educación secundaria costarricense. Uniciencia, 29(1), 42-57. doi: http://dx.doi.org/10.15359/ru.29-1.3

\section{(c) $(7)()_{\mathrm{EY}}$}

Esta obra está bajo una licencia de Creative Commons Reconocimiento-NoComercialSinObraDerivada 4.0 Internacional. 